09

\title{
Генерация аттосекундного импульса в газообразной среде атомов гелия, возбуждаемого полуцикловыми рентгеновскими импульсами
}

\author{
(c) Р.М. Архипов ${ }^{1,2,3}$, М.В. Архипов ${ }^{1,2}$, И. Бабушкин ${ }^{4,5}$, А.В. Пахомов ${ }^{2}$, Н.Н. Розанов ${ }^{2,3}$ \\ ${ }^{1}$ Санкт-Петербургский государственный университет, \\ 199034 Санкт-Петербург, Россия \\ 2 Университет ИТМО, \\ 197101 Санкт-Петербург, Россия \\ ${ }^{3}$ ФТИ им. А.Ф. Иофрфе, \\ 194021 Санкт-Петербург, Россия \\ ${ }^{4}$ Institute of Quantum Optics, Leibniz University Hannover, \\ 30167 Hannover, Germany \\ ${ }^{5}$ Cluster of Excellence PhoenixD (Photonics, Optics, and Engineering-Innovation across Disciplines), \\ 30167 Hannover, Germany \\ e-mail: arkhipovrostislav@gmail.com
}

Поступила в редакцию 13.12.2019 г.

В окончательной редакции 13.12.2019 г.

Принята к публикации 23.12.2019 г.

В традиционном подходе аттосекундные импульсы получаются с помощью генерации оптических гармоник высокого порядка при возбуждении различных сред фемтосекундным лазерным импульсом, центральная частота которого лежит в инфракрасном диапазоне. В работе теоретически рассматривается альтернативная возможность получения изолированного экстремально ультрафиолетового (XUV) аттосекундного импульса в тонком слое газа атомов гелия, возбуждаемого парой полуцикловых (квазиуниполярных) рентгеновских импульсов. Подход основан на явлении затухания свободной поляризации атомарной среды. Обсуждена связь рассматриваемых процессов с явлением сверхизлучения.

Ключевые слова: аттосекундные импульсы, рентгеновские импульсы, униполярные импульсы, атом гелия, сверхизлучение.

DOI: 10.21883/OS.2020.04.49205.346-19

\section{Введение}

Генерация аттосекундных импульсов $\left(1\right.$ as $\left.=10^{-18} \mathrm{~s}\right)$ длительностью порядка периода колебаний электромагнитной волны является одной из самых актуальных проблем современной оптики [1-4]. Аттосекундные импульсы позволяют эффективно управлять движением волновых пакетов электронов в атомах, молекулах и твердых телах [5-7]. Такие короткие импульсы света могут быть получены с помощью генерации гармоник высокого порядка (ГГВП) (high order harmonics generation, HHG) при взаимодействии инфракрасного фемтосекундного лазерного импульса с веществом [1-7]. Для теоретического описания процесса генерации аттосекундных импульсов используется полуклассическая модель ГГВП, известная в литературе как „трехступенчатая модель“(,three-step model“") $[1-4,8]$. Согласно этой модели, в основе процесса ГГВП лежат три основных этапа (процесса). На этапе 1, называемом ионизацией, электрон туннелирует через потенциальный барьер, созданный атомным потенциалом и лазерным полем. На этапе 2 электрон становится свободным и ускоряется под действием поля лазерного импульса. На последнем этапе 3 электрон рекомбинирует с родительским ионом. Во время этой стадии электрон испускает аттосекунд- ный импульс, спектр которого обычно лежит в экстремальном ультрафиолете (XUV) или рентгеновском диапазоне. Спектр такого сложного движения электрона представляет собой набор эквидистантных гармоник, удаленных друг от друга на величину центральной частоты импульса накачки. Этот спектр имеет почти плоский вид (плато) до некоторого значения частоты, которое называется частотой отсечки (cut off frequency). Когерентное сложение этих гармоник приводит к генерации последовательности аттосекундных импульсов [1-4]. Однако такие методы обладают низкой эффективностью, а также требуют громоздких и сложных экспериментальных установок. Поэтому поиск альтернативных методов получения предельно коротких импульсов является актуальной проблемой.

Впоследствии были предложены различные схемы ГГВГ для получения изолированных аттосекундных импульсов - метод стробирования (gating technique) $[9,10]$, метод с использованием двухчастотного лазерного поля накачки [11], а также при возбуждении атома водорода чирпированным лазерным импульсом [12] и т. д. Удается получить аттосекундные импульсы со стабилизированной фазой (carrier envelope phase, CEP), что необходимо для различных практических приложений, см., напримep, [12-14]. 
Недавно были получены и оптические аттосекундные импульсы [6]. Они были успешно применены для изучения динамики связанных электронов в газах [6]. В работах $[15,16]$ предложены способы получения одиночных, полуцикловых, оптических, аттосекундных импульсов, генерируемых при прохождении пучка быстрых электронов через мишень.

В работе [17] был предложен способ генерации полуциклового униполярного импульса при отражении одноциклового импульса от тонкой пленки металла или диэлектрика. Ключевой особенностью предложенного метода является использование линейного отражения и квазиодномерного распространения, которое реализуется для широких пучков. Данный метод можно использовать для генерации полуцикловых аттосекундных импульсов. Теория генерации предельно коротких импульсов в одномерных схемах была рассмотрена в работе [18].

Недавно было предложено использовать метод когерентного контроля низкочастотных колебаний в резонансной среде, возбуждаемой парой фемтосекундных импульсов, для генерации предельно коротких униполярных и терагерцовых импульсов [19-23]. Идея метода заключается в том, что первый фемтосекундный импульс возбуждает осцилляции низкочастотной поляризации среды, а второй импульс, который приходит спустя промежуток времени, равный половине периода колебаний низкочастотного осциллятора среды, останавливает эти колебания. В промежутке между импульсами в среде существует резонансная поляризация в течение некоторого времени порядка времени релаксации поляризации $T_{2}$ [24]. Она является источником субцикловых импульсов. Описанный метод было предложено использовать для генерации низкочастотных предельно коротких импульсов - униполярных импульсов [18-21] и терагерцовых импульсов $[18,22,23]$ при накачке фемтосекундными имульсами.

За счет метода ГГВП при ускорения свободных электронов в различных мишенях экспериментально удается получить рентгеновские аттосекундные импульсы, см., например, [25-27] и цитируемую там литературу. При ускорении свободных электронов излучаемые импульсы в принциипе могут иметь постоянную составляющую поля, т. е. являться униполярными [28,29]. Униполярные импульсы могут оказывать однонаправленное воздействие на заряженные частицы. Поэтому интерес к ним связан с возможностью их применения для эффективного контроля динамики волновых пакетов в веществе и ускорения заряженных частиц, см., например, [21,30-33]. О генерации униполярных импульсов и их применении см. также обзоры $[21,33]$ и приведенную там литературу.

Полуцикловые квазиуниполярные импульсы содержат всплеск поля одной полярности с большой амплитудой и „Хвост“ противоположной полярности с малой амплитудой $[6,15,16,21,30]$. Такие высокочастотные полуцикловые рентгеновкие импульсы, по-нашему мнению, также могут быть использованы для когерентного контроля

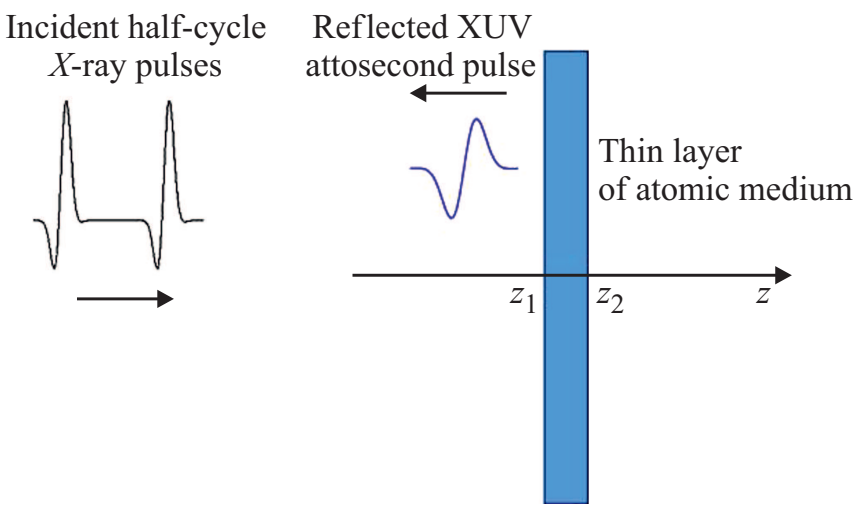

Рис. 1. Рассматриваемая система, возбуждаемая парой субцикловых рентгеновских импульсов, и генерируемый XUV или оптический одноцикловый аттосекундный импульс.

низкочастотных колебаний, лежащих, например, в оптической или ультрафиолетовой области спектра.

В настоящей работе изучается возможность генерации изолированного аттосекундного импульса в тонком слое атомарной двухуровневой среды, возбуждаемой парой рентгеновских полуцикловых импульсов. В качестве примера атомарной среды рассматриваются атомы гелия. Идея метода также основана на когерентном контроле колебаний поляризации в атомарной среде и использовании одномерной задачи распространения.

\section{Описание метода получения аттосекундного импульса и теоретическая модель}

Идея изучаемого метода иллюстрируется на рис. 1. Тонкий слой атомарной двухуровневой среды, частота перехода которой по отношению к рентгеновскому диапазону лежит в области более низких частот, располагается перпендикулярно оси $z$, между точками $z_{1}$ и $z_{2}$. Он возбуждается парой полуцикловых рентгеновских импульсов, имеющих плоский волновой фронт и распространяющихся вдоль оси $z$. Период следования импульсов равен половине периода собственных колебаний $T_{0} / 2$ на частоте резонансного перехода двухуровневой среды. Среда излучает одноцикловый аттосекундный импульс, который регистрируется в ближнем поле, что характерно для одномерной задачи распространения, справедливой на расстояниях, много меньших длины дифракции. Похожая схема получения ТГц и униполярных импульсов в трехмерной и одномерной задачах при возбуждении среды оптическими фемтосекундными импульсами была рассмотрена ранее в [18-23].

Поле падающих рентгеновских импульсов запишем в виде

$$
\begin{aligned}
E_{x}(t) & =E_{0 X} e^{\frac{-t^{2}}{\tau^{2}}} \cos \left(\omega_{x} t+\phi\right)+E_{0 X} e^{\frac{-\left(t-T_{d}\right)^{2}}{\tau^{2}}} \\
& \times \cos \left(\omega_{x}\left[t-T_{d}\right]+\phi\right) .
\end{aligned}
$$


Здесь $\omega_{x}$ - частота рентгеновских импульсов, $\phi-$ фаза (carrier envelope phase, CEP), $T_{d}$ - задержка между импульсами. Резонансную среду будем описывать в двухуровневом приближении с помощью формализма матрицы плотности. Взаимодействие субцикловых рентгеновских импульсов с двухуровневой резонансной средой описывается системой уравнений Максвелла-Блоха, которая имеет вид [34-35]

$$
\begin{gathered}
\frac{\partial \rho_{12}(z, t)}{\partial t}=\frac{-\rho_{12}(z, t)}{T_{2}}+i \omega_{0} \rho_{12}(z, t) \\
-\frac{i}{\hbar} d_{12} E(z, t) n(z, t), \\
\frac{\partial n_{12}(z, t)}{\partial t}=-\frac{n(z, t)-n_{0}(z, t)}{T_{1}} \\
+\frac{4}{\hbar} d_{12} E(z, t) \mathscr{T} \rho_{12}(z, t), \\
P(z, t)=2 N_{0} d_{12} \mathscr{R} \rho_{12}, \\
\frac{\partial^{2} E(z, t)}{\partial z^{2}}-\frac{1}{c^{2}} \frac{\partial^{2} E(z, t)}{\partial t^{2}}=\frac{4 \pi}{c^{2}} \frac{\partial^{2} P(z, t)}{\partial t^{2}} .
\end{gathered}
$$

В этих уравнениях $P-$ поляризация атомарной среды, $n \equiv \rho_{11}-\rho_{22}-$ разность заселенностей между основным и возбужденным состояниями двухуровневой системы, $\rho_{12}$ - недиагональный элемент матрицы плотности, $E$ - напряженность электрического поля с фиксированной линейной поляризацией, $N_{0}-$ концентрация частиц в слое, $c-$ скорость света в вакууме, $\hbar-$ приведенная постоянная Планка, $\omega_{0}-$ частота резонансного перехода среды $\left(\lambda_{0}=2 \pi c / \omega_{0}-\right.$ длина волны резонансного перехода), $n_{0}$ - разность заселенностей двух рабочих уровней при отсутствии электрического поля, $d_{12}-$ дипольный момент резонансного перехода, $T_{1}-$ время релаксации разности населенностей, $T_{2}-$ время релаксации поляризации. Ввиду малой длительности импульсов генерации и возбуждения пренебрежем конечностью времен релаксации $T_{1}$ и $T_{2}$. В газообразной среде их значения лежат в наносекундном диапазоне. Отметим, что система уравнений Максвелла-Блоха (2)-(5) записана без приближений медленно меняющихся амплитуд и вращающейся волны.

Другим приближением, принятым в нашей задаче, является одномерное приближение в волновом уравнении (5), справедливое для широких пучков на расстояних, меньших дифракционной длины. В рассматриваемом приближении аналитическое решение волнового уравнения (5) для отраженного средой поля генерации аттосекундного импульса имеет вид [17]

$$
E_{a}(z, t)=\frac{-2 \pi}{c} \int_{z_{1}}^{z_{2}} \frac{\partial}{\partial t} P\left(z^{\prime}, t-\frac{\left|z-z^{\prime}\right|}{c}\right) d z^{\prime} .
$$

Характерной особенностью одномерной задачи является то, что генерируемое поле пропорционально первой производной поляризации среды (скорости зарядов), а не их ускорению (второй производной поляризации), как в трехмерном случае [36].

Подходящим примером двухуровневой среды для иллюстрации идеи о генерации аттосекундного импульса, на наш взгляд, является атом гелия. Гелий обладает резонансным переходом с длиной волны примерно $60 \mathrm{~nm}$ из основного состояния $1^{1} S_{0}$ в первое возбужденное $2^{1} S_{0}[37,38]$. Остальные уровни гелия лежат относительно близко к первому возбужденному, и расстояние между уровнями быстро уменьшается с ростом главного квантового числа. Поэтому вклад остальных уровней в колебания поляризации будет заключаться лишь в наличии „хвоста“ малой амплитуды, который мы не учитываем. В этом смысле атом гелия является уникальным атомом, для которого в нашем случае двухуровневое приближение достаточно обосновано.

Пусть теперь среда возбуждается парой субцикловых рентгеновских импульсов (1) с периодом $T_{0} / 2$, равным половине периода резонансного перехода в среде (рис. $2, a$ ). Тогда первый импульс возбуждает колебания поляризации среды на частоте резонансного перехода среды, второй импульс останавливает их. Поляризация среды, согласно (4), пропорциональна вещественной части недиагонального элемента матрицы плотности $\mathscr{R} \rho_{12}$, которая на рис. $2, b$ изображена полуволной колебаний. Генерируемое поле аттосекундного импульса, согласно (6), пропорционально производной от поляризации среды и имеет вид одноциклового аттосекундного импульса. Оно изображено на рис. 2,c. Из рис. 2,c видно, что поле генерируемого излучения, пропроциональное производной поляризации, имеет вид одноциклового импульса длительностью около $T_{0} / 2 \sim 100$ as.

Рис. 2 был получен в результате численного решения системы уравнений Блоха (2)-(4) с полем рентгеновских импульсов (1). Параметры модели указаны в таблице.

Мы также проверили путем прямого решения уравнения Шредингера, что вероятность ионизации атома в таких ультракоротких импульсах очень мала, порядка $10^{-11}$, несмотря на то, что энергия фотонов больше потенциала ионизации. Более точные расчеты на основании решения уравнения Шредингера являются темой отдельного исследования и выходят за рамки настоящей работы.

\section{Упрощенная классическая теория генерации аттосекундного импульса в двухуровневой среде}

Для уяснения идеи генерации аттосекундного импульса обратимся к следующей упрощенной картине. Приведенные выше рассуждения легко описать математически, используя простую модель линейного гармонического осциллятора, которая вытекает из двухуровневой модели [35]. Нетрудно показать, что система уравнений Блоха (2),(3) может быть сведена к уравнениям для 
Параметры модели, используемые в расчетах

\begin{tabular}{l|c}
\hline $\begin{array}{l}\text { Амплитуда рентгеновских импульсов } \\
\text { возбуждения }\end{array}$ & $E_{0 X}=3 \cdot 10^{7} \mathrm{~V} / \mathrm{cm}$ \\
\hline Частота (длина волны) & $\omega_{x}=3.769 \cdot 10^{17} \mathrm{rad} / \mathrm{s}\left(\right.$ длина волны $\left.\lambda_{X}=5 \mathrm{~nm}\right)$ \\
\hline Длительность импульсов возбуждения & $\phi=3.1 \mathrm{as}$ \\
\hline СЕР & $\omega_{0}=3.141 \cdot 10^{16} \mathrm{rad} / \mathrm{s}\left(\lambda_{0}=60 \mathrm{~nm}\right)$ \\
\hline Частота перехода среды (длина волны перехода) & $d_{12}=1 \mathrm{D}$ \\
\hline Дипольный момент перехода & $T_{1}=T_{2}=\infty$ \\
\hline Времена релаксации & $N_{0}=10^{19} \mathrm{~cm}^{-3}$ \\
\hline Концентрация частиц в слое & $h=40 \mathrm{~nm}$
\end{tabular}
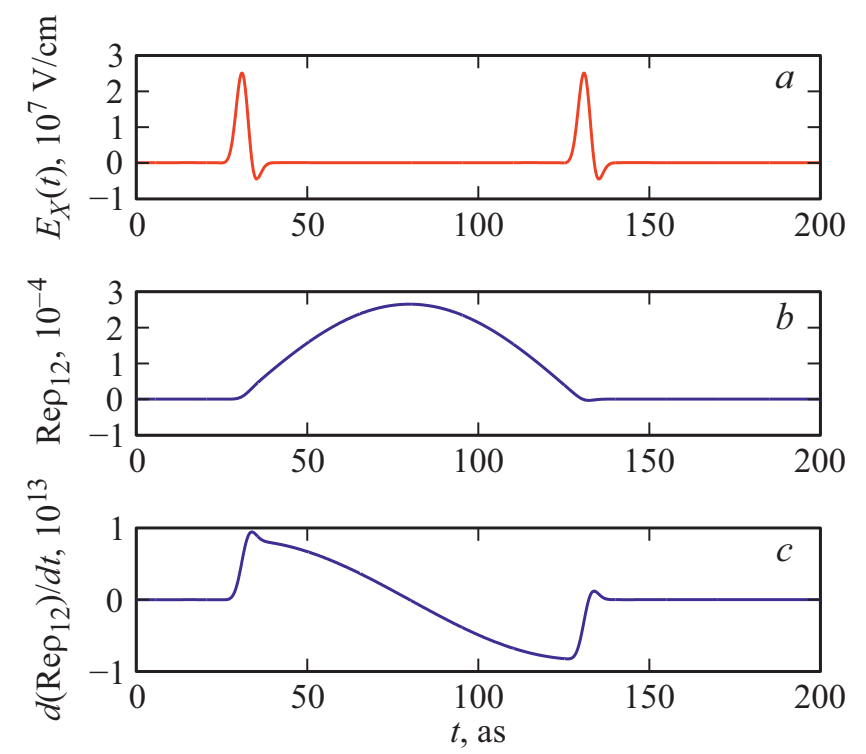

Рис. 2. (a) Полуцикловые рентгеновские импульсы возбуждения, следующие с периодом $T_{0} / 2\left(T_{0}=200 \mathrm{as}\right)$. (b) Поляризация единичного атома $\mathscr{R} \rho_{12},(c)$ Ее производная. Параметры импульсов возбуждения и среды приведены в таблице.

поляризации $P$ и разности населенностей $N=N_{0} n$ вида [35]

$$
\begin{aligned}
\ddot{P}+\frac{1}{T_{2}} \dot{P}+\omega_{0}^{2} P & =\frac{2 \omega_{0} d_{12}^{2}}{\hbar} E(t) N(t), \\
\dot{N}+\frac{N-N_{0}}{T_{1}} & =\frac{-2}{\hbar \omega_{0}} E(t) \dot{P}(t) .
\end{aligned}
$$

Пусть импульсы возбуждения (1) короче периода резонансного перехода в атоме $\left(\omega_{0} \tau \ll 1\right)$ и периода осцилляций Раби, что соответствует параметрам наших расчетов. В этом случае можно пренебречь изменением разности населенностей и в правой части уравнения (7) заменить $N(t)$ на равновесное значение $N_{0}$. Тогда вместо (7) имеем обычное уравнение для линейного гармонического осциллятора, которое в пренебрежении затуханием осциллятора, записывается в виде

$$
\ddot{P}+\omega_{0}^{2} P=\frac{2 \omega_{0} d_{12}^{2}}{\hbar} N_{0} E(t) .
$$

Приближенное аналитическое решение уравнения (9) при условии $\omega_{0} \tau \ll 1$ и при произвольной функции связи атома с полем было получено в работе [20]. Применим результат работы [20] к нашему случаю, отвечающему рис. 2 , когда система возбуждается парой квазиуниполярных импульсов с периодом $T_{0} / 2$. Тогда выражение для поляризации между импульсами имеет вид полуволны, которая может быть приближенно описана синусоидой

$$
P(t)=A \sin \omega_{0} t, \quad A \equiv \frac{2 d_{12}^{2}}{\hbar} N_{0} \int_{-\infty}^{\infty} E_{x}(t) \cos \omega_{0} t d t .
$$

Таким образом, первый импульс возбуждает колебания осциллятора на собственной частоте, описываемые формулой (10), а второй импульс останавливает эти колебания. Соответственно изображенная на рис. 2,c производная поляризация может быть описана одним циклом колебании косинусоиды

$$
\dot{P}=\omega_{0} A \cos \omega_{0} t,
$$

которая, согласно (6), отвечает одноцикловому импульсу генерации. Но приведенные в данном разделе рассуждения являются сильно упрощенными, поэтому в последующих разделах мы будем использовать численное решение системы уравнений Блоха (2), (3).

\section{Результаты численного моделирования}

Сказанное выше легко проиллюстрировать численным расчетом. Рассмотрим возбуждение тонкого слоя среды (рис. 1) с толщиной $h=40 \mathrm{~nm}$ парой рентгеновских импульсов с параметрами задачи, приведенными в таблице. 


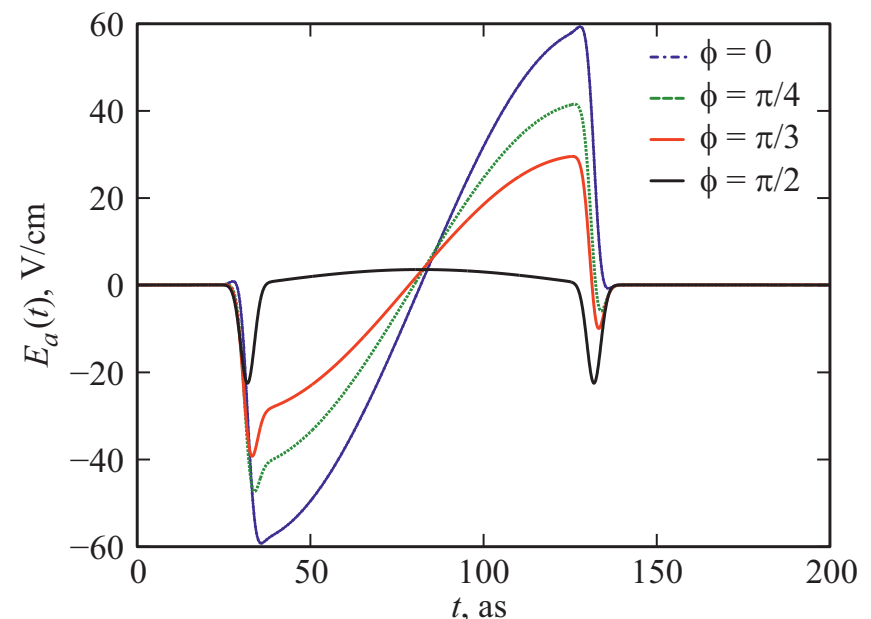

Рис. 3. Рассчитанные формы аттосекундных импульсов излучения $E_{a}$ при различных значениях СЕР накачки $\phi$. Параметры указаны в таблице.

Длину волны перехода возьмем $60 \mathrm{~nm}$, что соответствует переходу из основного состояния в первое возбужденное в атоме гелия [37,38]. Концентрация атомов гелия при комнатной температуре $p=N_{0} k T$ и нормальном атомосферном давлении $N_{0} \sim 10^{19} \mathrm{~cm}^{-3}$.

Для вычисления формы одноциклового аттосекундного импульса использовалась следующая процедура. Поляризация среды рассчитывалась с помощью численного решения системы уравнений Блоха (2)-(4) с электрическим полем возбуждающих импульсов в форме (1) и при параметрах задачи из таблицы. Электрическое поле генерации легко рассчитать по формуле (6). Толщина слоя $h$ бралась меньше длины волны излучения, поэтому при расчете импульса генерации в формуле (6) считалось, что простанственной зависимостью производной поляризации от координаты $z$ можно пренебречь. Поэтому электрическое поле генерации рассчитывалось по простой формуле, которая непосредственно следует из (4) и (6):

$$
E_{a}(t)=\frac{-4 \pi}{c} h N_{0} d_{12} \mathscr{R} \frac{\partial}{\partial t} \rho_{12}(t) .
$$

Рис. 3 иллюстрирует рассчитанные формы генерируемых XUV аттосекундных импульсов излучения при различных значениях фазы рентгеновских импульсов накачки СЕР $\phi$.

В предложенном методе важно, чтобы импульсы возбуждения имели квазиуниполярную субцикловую форму, как на рис. 2, $a$. Но форма импульса возбуждения зависит от его СЕР. Этим и объясняется зависимость формы аттосекундного импульса генерации от СЕР накачки на рис. 3 .

Согласно развитой полуклассической теории, энергетическая эффективность генерации при данных параметpax задачи составляет $10^{-11}$, длительность импульсов генерации порядка 100 as, амплитуда $60 \mathrm{~V} / \mathrm{cm}$. Для повышения эффективности надо рассматривать более толстые слои. В случае толстых слоев необходимо решать численно систему уравненияй Максвелла-Блоха, что выходит за рамки настоящей работы. При использовании длинных слоев, как показывают расчеты, приведенная выше идея генерациии одноциклового импульса остается также справедливой, однако форма импульса генерации может немного отличаться от одноцикловой [23].

Интересно отметить, что формой и длительностью аттосекундного импульса генерации можно управлять, меняя не только СЕР накачки, но и задержку между импульсами. Если взять задежку между импульсами $3 T_{0} / 2$, то 2-й импульс также остановит колебания поляризации, но длительность импульса генерации увеличится (рис. 4).

\section{Связь со сверхизлучением}

Рассматриваемые выше результаты были получены в условиях, когда длительность импульсов возбуждения и генерации много меньше времен релаксации $T_{1}$ и $T_{2}$ среды, значения которых для газов лежат в нс-диапазоне. Поэтому взаимодействие рентгеновских импульсов со средой является когерентным [24]. За временной промежуток между импульсами возбуждения фазовая когерентность среды не успевает разрушиться, и все атомы среды вносят одинаковый вклад в поляризацию среды (4) или (10). В этом смысле данная ситуация аналогична сверхизлучению - когерентному коллективному спонтанному излучению сфазированных диполей, образующих гигантский дипольный момент $[24,34]$. В рассматриваемом нами случае на рис. 2 осциллирующая поляризация излучает аттосекундный импульс в промежутке между импульсами возбуждения. В.Л. Гинзбург в заметке [39] „О природе спонтанного
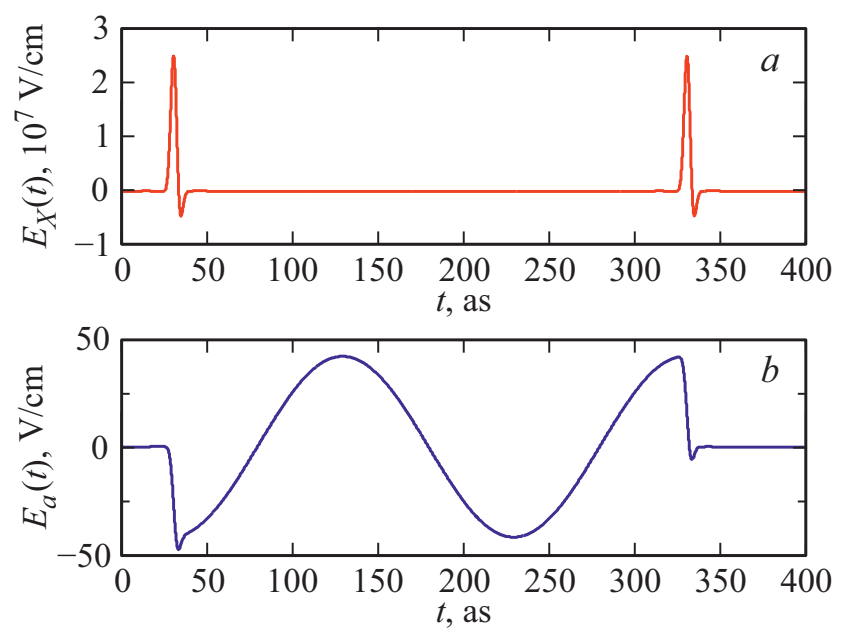

Рис. 4. (a) Пара рентгеновских импульсов возбуждения, следующих с задержкой $T_{d}=3 T_{0} / 2, \phi=\pi / 4,(b)$ аттосекундный импульс генерации. Остальные параметры такие же, как в таблице. 
излучения“ пришел к выводу, что спонтанное излучение имеет место не только в квантовой, но и в классической физике. Поэтому если под спонтанным излучением, соласно [39] (стр. 694), понимать „излучение системы, происходящее при отсутствии какого-либо внешнего (по отношению к системе) электромагнитного поля“', то расматриваемая нами ситуация имеет прямую аналогию со сверхизлучением - коллективным спонтанным излучением ансамбля атомов. Полное описание динамики сверхизлучения в данной системе выходит за рамки данной статьи, так как требует учета связи поля спонтанного излучения с атомами.

Интересно отметить, что использование эффекта сверхизлучения находит применения в генерации предельно коротких импульсов ТГц излучения в ансамбле квантовых точек [40]. Аналогичные коллективные когерентные процессы в комбинационно-активных молекулах, возбуждаемых фемтосекундными лазерными импульсами, могут также приводить к генерации коротких импульсов ТГц излучения с высокой эффективностью [22,23].

Рассмотренный в [40] способ характеризуется высокой эффективностью генерации, что, по-видимому, связано с тем, что при сверхизлучении все элементарные диполи излучают в фазе и тем самым вносят одинаковый вклад в осциллирующий дипольный момент среды. В нашей полуклассической модели оцененная эффективность довольно низкая из-за используемого приближение тонкого слоя. Однако уже грубая оценка по формуле (12) позволяет оценить энергетическую эффективность генерации порядка $10^{-4}$ для слоя толщиной $100 \mu \mathrm{m}$ (или массива тонких слоев среды, расположенных на расстоянии, кратном длине волны, друг от друга).

\section{Заключение}

В настоящей работе в одномерном приближении была рассмотрена возможность получения одноциклового аттосекундного импульса в двухуровневой атомной среде, возбуждаемой парой рентгеновских полуцикловых импульсов. В качестве примера атомной среды был рассмотрен атом гелия. Изученный метод основан на когерентном контроле колебаний поляризации среды с помощью квазиуниполярных, полуцикловых рентгеновских импульсов. Длительность импульса генерации составляет 100 as. Важной особенностью является простота управления формой и длительностью генерируемых импульсов с помощью изменения задержки между импульсами возбуждения и их СЕР. Данный метод позволяет получать аттосекундные импульсы в оптическом и экстремально ультрафиолетовом (XUV) диапазонах частот.

Обсуждена связь со сверхизлучением - колективным спонтанным излучением ансамбля диполей, когда все элементарные диполи излучают в фазе и вносят свой когерентный вклад в единый „гигантский“ дипольный момент среды [24,34,39]. Применение явления сверхизлучения для генерации коротких импульсов может приводить к высокой эффективности генерации ввиду коллективной и когерентной природы эффекта сверхизлучения $[22,23,40]$.

\section{Финансирование работы}

Исследования Р.М.А. и Н.Н.Р. выполнены при финансовой поддержке РФФИ в рамках научных проектов № 20-32-70049 (теория генерации аттосекундного импульса и численное моделирование) и 19-02-00312 (разделы, посвящённые упрощённой классической теории генерации аттосекундного импульса и связи со сверхизлучением). И.Б. благодарит за поддержку инновационный кластер PhoenixD (EXC 2122, Project Nr. 390833453) и Deutsche Forschungsgemeinschaft (DFG), проект BА4156/4-2.

\section{Конфликт интересов}

Авторы заявляют, что у них нет конфликта интересов.

\section{Список литературы}

[1] Corkum P.B., Krausz F. // Nature Physics. 2007. V. 3. N 6. P. 381.

[2] Krausz F., Ivanov M. // Rev. Mod. Phys. 2009. V. 81. P. 163.

[3] Calegari F. etal. // J. Physics B: Atomic, Molecular and Optical Physics. 2016. V. 49. N 6. P. 062001.

[4] Стрелков В.В., Платоненко В.Т., Стержантов А.Ф., Рябикин М.Ю. // УФН. 2016. Т. 186. № 5. С. 449-470; Strelkov V.V., Platonenko V.T., Sterzhantov A.F., Ryabikin M.Yu. // Phys. Usp. 2016. V. 59. N 5. P. 425-445.

[5] Ramasesha K., Leone S.R., Neumark D.M. // Annu. Rev. Phys. Chem. 2016. V. 67. P. 41.

[6] Hassan M.T., Luu T.T., Moulet A., Raskazovskaya O. etal. // Nature. 2016. V. 530. P. 66.

[7] Nisoli M., Decleva P., Calegari F., Palacios A., Martin F. // Chemical Reviews. 2017. V. 117. N 16. P. 10760-10825.

[8] Corkum P.B. // Phys. Rev. Lett. 1993. V. 71. P. 1994-1997.

[9] Sola I.J., Mével E., Elouga L., Constant E., Strelkov V. et al. // Nature Physics. 2006. V. 2 (5), P. 319.

[10] Chen G., Cunningham E., Chang Z. // J. Modern Optics. 2017. V. 64. P. 952-959.

[11] Tai H., Li F., Wang Z. // J. Modern Optics. 2016. V. 63 (12). P. 1166-1173.

[12] Mauritsson J., Johnsson P., Gustafsson E., L'Huillier A., Schafer K.J., Gaarde M.B. // Phys. Rev. Lett. 2006. V. 97. P. 013001.

[13] Tibai Z., Tóth G., Mechler M.I., Fülöp J.A., Almási G., Hebling J. // Phys. Rev. Lett. 2014. V. 113. N 10. P. 104801.

[14] Navid H.A., Aghbolaghi R., Yarali Z. // J. Modern Optics. 2019. V. 66. N 17. P. 1744-1753.

[15] Wu H.-C., Meyer-ter-Vehn J. // Nature Photon. 2012. V. 6. P. 304.

[16] Xu J., Shen B., Zhang X. etal. // Sci. Rep. 2018. V. 8. P. 2669. 
[17] Arkhipov M.V., Arkhipov R.M., Pakhomov A.V., Babushkin I.V., Demircan A., Morgner U., Rosanov N.N. // Opt. Lett. 2017. V. 42. N 11. P. 2189-2192.

[18] Пахомов А.В., Архипов Р.М., Архипов М.В, Бабушкин И., Розанов Н.Н. // Опт. и спектр. 2017. Т. 123. В. 6. C. 901-906; Pakhomov A.V., Arkhipov R.M., Arkhipov M.V., Babushkin I., Rosanov N.N. // Opt. Spectrosc. 2017. V. 123. N 6. P. 913-917.

[19] Arkhipov R.M., Arkhipov M.V., Belov P.A., Tolmachev Yu.A., Babushkin I. // Las. Phys. Lett. 2016. V. 13. P. 046001.

[20] Pakhomov A.V., Arkhipov R.M., Babushkin I.V., Arkhipov M.V., Tolmachev Yu.A., Rosanov N.N. // Phys. Rev. A. 2017. V. 95. P. 013804.

[21] Архипов Р.М., Пахомов А.В., Архипов М.В., Бабушкин И., Толмачев Ю.А., Розанов Н.Н. // Письма в ЖЭТФ. 2017. T. 105. № 6. C. 388-400; Arkhipov R.M., Pakhomov A.V., Babushkin I., Tolmachev Yu.A., Rosanov N.N. // JETP Lett. 2017. V. 105. N 6. P. 408-418.

[22] Pakhomov A.V., Arkhipov R.M., Arkhipov M.V., Demircan A., Morgner U., Rosanov N.N. // Sci. Rep. 2019. V. 9. N 1. P. 7444.

[23] Arkhipov R.M., Pakhomov A.V., Arkhipov M.V., Demircan A., Morgner U., Rosanov N.N. // Phys. Rev. A., в печати (arXiv preprint arXiv:1907.11488).

[24] Аллен Л., Эберли Дж. Оптический резонанс и двухуровневые атомы. М.: Мир, 1978; Allen L., Eberly J.H. Optical Resonance and Two-level Atoms. N. Y.: Wiley, 1975.

[25] Ishii N., Kaneshima K., Kitano K., Kanai T., Watanabe S., Itatani J. // Nature Commun. 2014. V. 5. P. 3331.

[26] Chini M., Zhao K., Chang Z. // Nature Photonics. 2014. V. 8 N 3. P. 178.

[27] Chang Z., Corkum P.B., Leone S.R. // JOSA B. 2016. V. 33. N 6. P. 1081-1097.

[28] Бессонов Е.Г. // ЖЭТФ. 1981. Т. 80. № 3. С. 852-858; Bessonov E.G. // Sov. Phys. JETP. 1981. V. 53. N 3. P. 433-436.

[29] Бессонов Е.Г. // Квант. электрон. 1992. Т. 19. № 1. С. 35-39; Bessonov E.G. // Sov. J. Quant. Electron. 1992. V. 22. N 1. P. 27-31.

[30] Arkhipov R.M., Arkhipov M.V., Babushkin I.V., Demircan A., Morgner U., Rosanov N.N. // Opt. Lett. 2019. V. 44. N 5. P. 1202.

[31] Архипов Р.М., Архипов М.В., Пахомов А.В., Розанов Н.Н. // Опт. и спектр. 2020. Т. 128. № 1. C. 106-109; Arkhipov R.M., Arkhipov M.V, Pakhomov A.V., Rosanov N.N. // Opt. Spectrosc. 2020, V. 128. N 1. P. $102-105$.

[32] Розанов Н.Н., Высотина Н.В. // ЖЭТФ. 2020. Т. 157. № 1. C. 63-66.

[33] Архипов Р.М., Архипов М.В., Шимко А.А., Пахомов А.В., Розанов Н.Н. // Письма в ЖЭТФ. 2019. Т. 110. № 1. C. 9-20; Arkhipov R.M., Arkhipov M.V., Shimko A.A., Pakhomov A.V., Rosanov N.N. // JETP Lett. 2019. V. 110. N 1. P. $15-24$.

[34] Ярив А. Квантовая электроника. М.: Сов. Радио, 1980; Yariv A. Quantum electronics.Wiley, 1989.

[35] Ахманов С.А., Никитин С.Ю. Физическая оптика. М.: Наука, 2004; Akhmanov S.A., Nikitin S.Y. Physical Optics. Clarendon Press, 1997.

[36] Борн М., Воль $\varnothing$ Э. Основы оптики. Изд. 2-е. Перевод с англ. М.: Главная редакция физико-математической литературы. Наука, 1973; Born M., Wolf E. Principles of Optics: Eectromagnetic Theory of Propagation, Interference and Diffraction of Light. Elsevier, 2013.
[37] Собельман И.И. Введение в теорию атомных спектров. М.: Наука, 1977.

[38] Фриш С.Э. Оптические спектры атомов. Государственное издательство физико-математической литературы, М.-Л., 1963.

[39] Гинзбург В.Л. // УФН. 1983. Т. 140. С. 687-698; Ginzburg V.L. // Sov. Phys. Usp. 1983. V. 26. P. 713-719.

[40] Zhang W.D., Brown E.R., Mingardi A., Mirin R.P., Jahed N., Saeedkia D. // Appl. Sci. 2019. V. 9. N 15. P. 3014. 